\title{
Criação e implantação do IEA de São Carlos
}

\author{
SÉRGIO MASCARENHAS e YVONNE PRIMERANO MASCARENHAS
}

\section{Criação}

GÊNESE da criação do Instituto de Estudos Avançados (IEA) da USP de
São Carlos passa pelo estágio de Sérgio Mascarenhas como fellow no Ins-
titute for Advanced Study (IAS), Princeton, Estados Unidos, em 1996, e com sua participação durante cerca de 12 anos como diretor do Curso de Biofísica e Fisica Médica no International Center for Theorethical Physics (ICTP), em Trieste, Itália, a convite de Abdus Salam, Prêmio Nobel de Física 1979, até o seu falecimento, em 1996. Sérgio Mascarenhas se convenceu de que tanto o modelo do ICTP como o do IAS poderiam se aplicar para a América Latina com grandes vantagens em face da semelhança de idioma, problemas e condições culturais, sociopolíticas e econômicas dos países da região. Ainda nos Estado Unidos, contatou várias agências, tais como as fundações Ford, Guggenheim, Rockefeller, em busca de apoio ao que denominou Programa de Inovação e Estudos para a América Latina (Piepal).

Uma resposta positiva foi obtida na Ford Foundation por meio do forte apoio da Dra. Joan Dassin, diretora de Programas para América Latina e Caribe, daquela instituição. A Dra. Dassin conhecia bem o Brasil, onde fora diretora de programa da Ford, além de imensamente identificada culturalmente com o país. Ao voltar ao Brasil, tendo conseguido um grant inicial da Ford, Sérgio Mascarenhas procurou o Prof. Flávio Fava de Morais, então reitor da USP, propondolhe a implantação do Piepal na USP-São Carlos. O Prof. Fava então, com o seu característico entusiasmo e visão, propôs que o Piepal fosse criado no âmbito de um novo polo do IEA da USP em São Carlos. Era um presente em bandeja de prata!

Contatos posteriores com o diretor do já existente IEA do campus da USP de São Paulo, Prof. Umberto Giuseppe Cordani, levaram ao apoio e estímulo de seu diretor à criação do polo de São Carlos. O mesmo apoio foi dado por todos os diretores das unidades locais: Instituto de Física, Instituto de Química, Instituto de Matemática e Computação e Escola de Engenharia de São Carlos, que constituíam o Conselho do Campus. Como consequência de todos esses apoios, foi conseguida a institucionalização do acordo com a Ford Foundation, tendo todos os diretores do campus acordado em priorizar a construção de um pequeno espaço físico para o IEA-USP-SC, que seria ocupado juntamente com a Rádio USP. Nessa fase foi fundamental o apoio da prefeitura do campus de São 
Carlos, que abrigou o IEA-SC provisoriamente até o término da construção do espaço de cerca de 100 metros quadrados e que, embora exíguo, deu corpo e alma ao IEA-USP-SC, abrigando um pequeno auditório para 40 lugares, quatro pequenas salas para secretaria, biblioteca e professores.

\section{Atividades iniciais}

Os recursos fornecidos pela Ford Foundation permitiram realizar três atividades priorizadas de acordo com o possível impacto em âmbito regional.

A primeira atividade, de caráter educacional, envolveu a importante contribuição do Centro de Difusão Científica e Cultural (CDCC), órgão interunidades do campus de São Carlos da USP, que orientou as atividades educacionais e pedagógicas do projeto, tanto no Brasil como na Argentina, na área de ensino de ciências para alunos do curso fundamental, abordando o importante tema Educação Ambiental. O Programa Piepal (Ford Foundation e Ieasc-USP - São Carlos, incluiu o Programa Educar dentro dos Projetos de Colaboração Mútua firmados em 7 de setembro de 2000.

Instituições participantes: La Casa de las Ciencias (UNC em Córdoba) Centro de Divulgação Científica e Cultural (USP - São Carlos) e Escolas Estaduais de ambos os países. A metodologia usada já estava bem desenvolvida no CDCC e foi transferida e adaptada às condições sociais e pedagógicas da escola congênere parceira de Córdoba, Argentina, respectivamente, Escola Estadual Bispo Dom Gastão em São Carlos e Escuela Gobernador Alvarez em Córdoba.

Ao final dessa colaboração, que se estendeu por cerca de 18 meses, foi produzido um livro on-line (português e espanhol), O estudo de bacias hidrográficas: uma estratégia para educação ambiental, assim como uma versão impressa.

Durante a realização do projeto, aconteceram o I e o II Congreso Iberoamericano de Educación em Ciencias Experimentales, respectivamente em São Carlos e Córdoba, com a participação de vários membros participantes brasileiros e argentinos. Nessas ocasiões, foi feita a divulgação dos resultados até aquela época obtidos e incorporação de novas atividades. A seguir, alguns momentos dos congressos e membros participantes.

- I Workshop Regional - $1^{\circ}$ a 5 de março de 1999 - Projeto: "Educação e Sociedade: Melhoria do Ensino Básico de Ciências na América Latina" - Programa: Piepal - Apoio Ford Foundation. Os participantes do workshop foram os professores Horácio Panepucci (diretor do IFSC/USP), Regina Helena P. Francisco (IQSC/USP), Eduardo Miguel González (coordenador do Grupo de Córdoba), Dietrich Schiel (diretor do CDCC/USP), Sérgio Mascarenhas (diretor do Piepal e coordenador do Ieasc/USP) e João Batista Gasparini (representante do delegado de Ensino de São Carlos). Da mesa-redonda "O papel da Universidade na Transformação da Educação" participaram os professores José Alberto Riveros (presidente do Consejo Directivo del Conicor), Myriam Krasilchik (diretora da Faculdade de Educação da USP), Jorge Daniel Pérez (ministro de Educación y Cultura de la Provincia de Córdoba), José Galizia 
Tundisi (secretário de Ciência e Tecnologia de São Carlos) e Yvonne Primerano Mascarenhas (vice-coordenadora do IEA).

No workshop, foram realizadas oficinas de Intercâmbio de Experiências Educacionais, como a "Física - Experimentoteca" - Hilton Koiti Sato e o Grupo de Córdoba: com os professores Eduardo Miguel González e Adriana Ferreyra.

- II Congresso Iberoamericano de Educação em Ciências Experimentais, realizado em Córdoba, Argentina, nos dias 5 a 8 de setembro de 2000. Realização da Universidade Nacional de Córdoba, Universidade Nacional de Rio Quarto e Universidade de Alcalá, Espanha.

No congresso, tivemos a conferência do professor Alberto Sasson: "O ensino das ciências no início do século XXI: desafios e perspectivas", e a conferência do professor Daniel Gil Pérez, "Ciências para todos? Alguns obstáculos e medidas a adotar à luz da investigação educativa”.

Foram realizadas as seguintes mesas-redondas: "Canais de ligação entre a Universidade e o meio" com a participação de Carlos Debandi (Agencia Córdoba Ciencia, Argentina), Daniel Lescano (Trasyt. UNC, Argentina), Silvia Etchegaray (UNRC, Argentina), Virginia Vargas (UMSS, Bolívia), Adriana Ferreyra (UNC, Argentina) e Yvonne P. Mascarenhas (Ieasc - USP, Brasil); e "Divulgação e popularização" com Maria Teresa Ferrero de Roqué (UNC), Miguel Ángel Gómez Crespo (Madri/Espanha), Graciela Merino (UNLP, Argentina), Alicia Baraibar (MEC, Uruguai) e Dietrich Schiel (CDCC/USP - Brasil).

Ao final dessa colaboração, que se estendeu por cerca de três anos, foi produzido um livro on-line (português e espanhol): Educação e sociedade: methoria do ensino básico de ciências na América Latina (Caso Piloto - São Carlos (Brasil) - Córdoba (Argentina)), além de O estudo de bacias hidrográficas: uma estratégia para educação ambiental, organizado e editado por Dietrich Schiel, Sérgio Mascarenhas, Nora Valeiras e Silvia A. M. dos Santos.

\section{Considerações finais}

As atividades foram realizadas com ampla troca de mensagens entre alunos e docentes de ambas as instituições via internet, visitas de pesquisadores argentinos de Córdoba a São Carlos, e vice-versa dos pesquisadores brasileiros a Córdoba, gerando oportunidades de experiências muito enriquecedoras. A criação desses vínculos por meio de intercâmbios entre alunos, professores e autoridades governamentais dos dois países da America Latina mostra claramente o quanto pode ser feito nesse tipo de integração latino-americana, por meio da educação científica e cultural de crianças e jovens.

Realizou-se um programa de ensino e divulgação científica para uma rede de Centros e Museus de Ciências na América Latina em parceria com a Estação Ciência de São Paulo, à época dirigida pelos professores Ernesto Hamburger e Silvério Crestana.

Conseguiu-se reunir em um congresso praticamente todos os mais importantes Centros e Museus de Ciências da América Latina, e publicou-se um 
alentado volume com todas as contribuições dos participantes, levando à proposta de um Projeto Integrado de Centros e Museus de Ciências para a América Latina a ser apresentado ao BID e aos órgãos locais de financiamento. Cremos que essa foi uma iniciativa histórica e duradoura que pode ser avaliada pelo crescimento da rede de centros e museus de ciências na América Latina desde a sua realização. Dessas atividades resultou a publicação do livro Centros e museus de Ciência - visões e experiências, organização de Silvério Crestana, Mirian Goldman de Castro e Gilson R. de M. Pereira.

A segunda atividade foi escolhida em decorrência de experiência anterior de vários pesquisadores em São Carlos, tanto da USP como da UFSCar, entre os quais Yvonne P. Mascarenhas, com experiência na área de cerâmicas tecnológicas, com projetos apoiado pela Petrobras, pelo CNPq e pela Fapesp. Outro fator importante foi a existência em São Carlos do Parque Tecnológico de São Carlos (ParqTec), dirigido por Silvio Rosa, professor aposentado do IFSC, que tinha uma boa visão do estágio de desenvolvimento regional da indústria de cerâmicas. Na mesma época, elaborava-se um projeto temático denominado "Materiais ferroelétricos: fenomenologia, propriedades e caracterização", coordenado por Yvonne P. Mascarenhas, que envolvia pesquisadores da USP/SC e da UFSCar, que foi posteriormente apoiado pela Fapesp. Levando em conta todas essas razões, foi tomada a decisão de realizar um workshop sobre materiais cerâmicos usando os recursos do Grant da Ford Foundation. Esse workshop contou com a participação de pesquisadores de São Paulo, do Paraná, de Goiás e de Minas Gerais.

\section{Estruturação atual}

Ultrapassada essa primeira fase do projeto Ford-Piepal-IEA-USP-SC, organiza-se o IEA-USP-SC de forma mais permanente por meio de Grupos de Trabalho (GT) com missões bem planejadas, e respectivos Planos de Ação Temáticos. Cada GT foi formado contando com um coordenador que poderia agregar outros pesquisadores da USP ou de outras instituições e buscar recursos nas agências de fomento públicas e/ou privadas.

Devemos, entretanto, citar que a institucionalização do IEA de São Carlos somente se deu em 2009 com a correspondente modificação do Estatuto da USP e do Regimento do IEA, dando-se, a partir de então, a denominação de Polos aos IEA dos campi de São Carlos, Ribeirão Preto e Piracicaba, e que seriam administrados por um coordenador. Foi então indicado o Prof. Roberto Faria, professor titular e ex-diretor do IFSC, para exercer a coordenadoria do Polo, após serem ouvidos todos os diretores de unidades do campus de São Carlos, que encaminharam uma lista de nomes ao Conselho Deliberativo do IEA, que, por sua vez, formulou uma lista tríplice que foi encaminhada à reitoria da USP. O Prof. Sérgio Mascarenhas foi então indicado pelo atual coordenador como vice-coordenador de Projetos, e a Profa. Yvonne P. Mascarenhas, vicecoordenadora para Assuntos Administrativos. 
As necessidades de serviços administrativos do IEA-SC são atendidas desde o início por duas secretarias já concursadas, nível técnico, o que permite o funcionamento do IEA-USP-SC cumprindo todas as exigências regimentais, bem como aquelas decorrentes das atividades dos GT com seus membros, redes e órgãos públicos e privados, incluindo relatórios, prestação de contas, comunicação postal e informatizada. Já as atividades financeiras com recursos USP a serem desempenhadas pelo IEA-SC foram sediadas na prefeitura do campus, atual Coordenadoria Administrativa do campus de São Carlos.

Os sucessivos diretores do IEA-USP sempre tiveram uma atitude de apoio e estimulo, assim como todos os reitores que passaram na Direção da Universidade em todo esse período de 1997 até o presente.

Levando em conta o potencial em recursos humanos existentes nos campi das duas Universidade (USP e UFSCar), nos dois Centros de Pesquisa da Embrapa e em algumas empresas de base tecnológica existentes na região de São Carlos, foram estabelecidos, mesmo antes da institucionalização, vários Grupos de Trabalho que, por meio de eventos esporádicos, tais como realização de conferências, seminários e workshops, têm contribuído para o levantamento e a discussão de tópicos abrangentes de interesse geral no campus de São Carlos da USP, ou mesmo estabelecendo programas de pesquisa de médio ou longo prazo. Foi então decidido abordar temas de importância para a solução dos problemas crônicos da América Latina. Entre esses problemas, avultam o da Educação de Nível Fundamental e Médio, cuja gravidade é muito bem atestada pelos fracos resultados obtidos em avaliações realizadas por organismos internacionais, e a Agricultura e Agronegócio do qual dependem nossa economia (um terço do PIB) e saúde do provo brasileiro pelo acesso a alimentos de boa qualidade.

\section{Grupos de trabalbo}

\section{Coordenação de projetos do IEA-USP-SC}

Missão: estruturar, apoiar e dirigir as ações decorrentes de projetos de pesquisa e relacionamento com instituições municipais, estaduais e federais de fomento, bem como os contatos internacionais - coordenador inicial de 1997 a 2011: Prof. Sérgio Mascarenhas.

\section{Rede de Inovação e Prospecção Tecnológica para o Agronegócio (Ripa) e} GT - coordenação de Silvio Crestana e Paulo Cruvinel, ambos pesquisadores da CNPDIA/ Embrapa.

Missão: estruturar Programas Estratégicos para o Agronegócio Brasileiro nas cinco regiões do país (Sul, Sudeste, Centro-Oeste, Nordeste e Norte).

O projeto foi desenvolvido com o apoio não apenas da Embrapa, mas principalmente da Finep, que financiou o programa por quatro anos. Esse GT teve repercussão profunda e, por meio da metodologia Delphi de Planejamento Cooperativo, conseguiu incorporar resultados aos planos de ação do governo. O Projeto Ripa, de âmbito nacional, completou seus objetivos gerais nas cinco regiões do Brasil, conforme pode ser visto no site www.ripa.com.br. A contribui- 
ção desse projeto foi tal que movimentou mais de mil pesquisadores, dirigentes empresariais, políticos, nos três níveis (executivo, estadual e municipal), ONG e outros representantes da sociedade brasileira. O produto final é um banco de dados de livre acesso com valiosas informações para os mais variados setores do agronegócio brasileiro.

O modelo de prospecção também foi aplicado em São Carlos, em parceria com o Sindicato Rural da cidade, e resultou em um trabalho publicado pela Embrapa Instrumentação Agropecuária e abordou as cadeias produtivas da apicultura, da bovinocultura leiteira e da caprinocultura.

\section{Difusão científica como apoio à Educação}

Missão: Melhorar a qualidade do ensino e aprendizagem nas escolas públicas mediante a interação Universidade-EP e uso de recursos multimídia.

Coordenação: Profa. Yvonne P. Mascarenhas com apoio importante do CDCC.

Metodologia:

a) Incentivo do uso de recursos de informática nas escolas públicas de ensino fundamental e médio por meio do treinamento de professores e alunos e apoio a atividades realizadas com apoio de professores universitários, professores do ensino fundamental e médio, alunos de cursos de graduação, tanto pesquisando na internet como realizando experimentos.

b) Criação de uma Agência de Difusão Científica denominada CienciaWeb, onde muitas matérias de interesse constituídas por objetos produzidos para esse público-alvo são depositadas no portal no endereço www.cienciaweb. com.br. Tais objetos são produzidos com apoio de jornalista profissional, professores universitários e das escolas públicas, estudantes de jornalismo, de imagem e som, de ciências da computação e de licenciatura em matemática e ciências exatas.

c) Utilização dos recursos existentes na Agência CienciaWeb como veículo de difusão dos resultados mais interessantes para o nosso público-alvo e população em geral obtidos pelos pesquisadores dos INCT de Eletrônica Orgânica do IFSC/USP, Sistemas Embarcados Críticos sediado no ICMC/USP/SC e da rede NanoBioTec-Brasil da Capes, em particular a sub-rede NanoBioMed sediada no IFSC/USP.

d) Realização de dois seminários sobre Difusão Científica com duração de dois dias. O primeiro realizado em outubro de 2010 sobre o tema "Uso da difusão científica como recurso educacional", com a participação de profissionais da área e educadores, e o segundo sobre o tema "Desafios da comunicação científica", a ser realizado em outubro de 2011. O I Seminário do CienciaWeb sobre o tema "Difusão científica como fonte para educação" teve por objetivo reforçar a importância da divulgação científica em nossa sociedade, com vistas a contribuir para a melhoria e atualização do ensino de ciências, para a difusão 
do tema nos meios de comunicação em nossa região, assim como para estimular a participação popular acerca de temas comuns ao dia a dia da educação. $\mathrm{O}$ seminário teve 143 inscrições, 90 presentes, e foi transmitido ao vivo com 142 acessos pela internet. A programação constou de palestras, mesas-redondas e workshops direcionados. Mais informações podem ser encontradas no site www. cienciaweb.com.br/seminario.

e) Série de palestras destinadas a professores de química e público em geral em comemoração do Ano Internacional da Química (apoio CNPq).

Os resultados desses trabalhos são bastante animadores, com boa aceitação pelas escolas parceiras do projeto, aumento de interesse dos professores e dos alunos, comunicações em congresso, publicações e repercussões na imprensa, rádio e TV regionais. Listamos a seguir publicações e resultados recentes apresentados em congressos:

(a) XVI CONGRESS World Association for Educational Research.Monterrey - México - 31 junho a 4 julho, 2010, título do colóquio: " $\mathrm{O}$ olhar da pesquisa em educação sobre o lugar e o papel dos saberes escolares disciplinares nas práticas docentes: conseqüências para o ensino e para a formação de professores; título da comunicação: "The use of new technologies in the daily school practice: learning motivation of students and teachers", de Neucideia Aparecida S. Colnago, Marina M. Massocco, Yvonne P. Mascarenhas, Rita C. BortolettoSantos.

(b) ED-Media 2011 World Conference on Educational Multimedia, Hypermedia and Telecomunication. Lisbon, Portugal, June 27-July 1, 2011. Título da comunicação: "Use of Multimedia to Improve Brazilian Public School Students' Interest in Science", de Yvonne P. Mascarenhas, Neucideia A. Colnago, Rita C. Bortoletto-Santos, Valda Rocha.

(c) Colnago, N. A. S.; Bortoletto-Santos, R. C., Mascarenhas, Y. P. (2010). Almanaque digital-Nosso Céu. Disponível em: http://muraldaescola.wordpress.com/2010/10/29/almanaque-digital-\%E2\%80\%9Cnossoceu\%E2\%80\%9D/\#more-554;

(d) Colnago, N. A. S.; Bortoletto-Santos, R. C.; Mascarenhas, Y. P. (2011). Almanaque digital - Conhecendo e difundindo os benefícios do mel. http://muraldaescola.files.wordpress.com/2011/02/cd-mel-final.pdf.

(e) Colnago, N. A. S.; Bortoletto-Santos, R. C.; Soares S. R. B.; Cordeiro A. P. M.; Almeida R. S.; Mascarenhas, Y. P. (2011). Almanaque Digital II Multidisciplinar: Sustentabilidade-Alternativa para o Futuro Encontradas no Passado. Disponível em: http://muraldaescola.files.wordpress.com/201 1/02/ cd-sustentabilidade-final.pdf.

(f) Rolfsen Belda, F.; Mascarenhas, Y. P. Aprendizagem em ambientes e comunidades televisivas: uma experiência-piloto em colégios da rede estadual de ensino de São Carlos (SP). InterScience Place, v.15, p.95-116, 2010. 


\section{Universidade empresas}

Coordenado pelo Prof. João Fernando Gomes de Oliveira, professor titular de Easc, atual diretor do Instituto de Pesquisas Tecnológicas (IPT) e coordenador do Instituto Nacional de Ciência e Tecnologia do MCT denominado Instituto Fábrica do Milênio, sediado no Departamento de Produção da EESC/USP. Os objetivos gerais do Instituto Fábrica do Milênio (IFM) podem ser entendidos como a proposição, o desenvolvimento e a disseminação de mecanismos para o aumento da competitividade e do conhecimento científico e tecnológico das indústrias de manufatura, especialmente as de bens de capital, instaladas no país. Tais objetivos podem inicialmente parecer muito abrangentes e pretensiosos. Entretanto, o conjunto de instituições e projetos envolvidos forma uma massa crítica que o capacita a desenvolver um espectro de pesquisas (que abrangem desde o desenvolvimento de tecnologias industriais básicas até elementos de gestão) que podem, se organizadas de forma integrada, contribuir efetivamente para o desenvolvimento das indústrias brasileiras. Dessa forma, o papel principal da coordenação do IFM é gerir a integração desses projetos e promover a difusão de seus resultados. Sua interação com o Polo do IEA/SC tem se dado por meio da organização de palestras proferidas por especialistas com a finalidade de discutir e divulgar os temas de sua área de pesquisa.

\section{Meio ambiente}

Sob a coordenação do Prof. José Galizia Tundisi, professor titular aposentado da EESC e diretor do Instituto Internacional de Ecologia, pesquisador brasileiro de renome internacional e que tem grande interesse na área educacional. Em sua interação com o IEA, salientamos que realizou não apenas atividades de pesquisa e inovação na área, mas também cooperou ativamente no programa de Bacia. Este ano colaborou com o GT de Difusão Científica como Apoio à Educação proferindo palestra no programa de comemorações "CienciaWeb no Ano Internacional da Química" subordinada ao tema "A química e a vida no Planeta Terra: implicações da contaminação dos recursos hídricos na qualidade da água e na biodiversidade".

\section{Aeronáutica espacial}

Coordenado pelo Prof. Dr. Fernando Martini Catalano, professor titular do Depto. de Engenharia Aeronáutica/EESC/USP, esse GT apoiou acordos de cooperação com o CTA de São José dos Campos mediante convênio com o Departamento de Aeronáutica da EESC para construção de um túnel ultrassônico em cooperação com o Instituto de Estudos Avançados do CTA. Foi atraído para esse GT o astronauta brasileiro Marcos Pontes, que se incorporou às atividades do GT.

\section{Tecnologia da informação}

Sob a coordenação do Prof. José Carlos Maldonado, professor titular do ICMC/USP e atual seu diretor, e coordenador do Instituto Nacional de Ciência e Tecnologia em Sistemas Embarcados Críticos (INCT-SEC), e do Prof. Dr. 
Edson dos Santos Moreira, professor titular do ICMC;USP. Esse GT foi instalado com o objetivo do elevar o nível de conhecimento, competência e qualidade no país sobre o desenvolvimento de sistemas embarcados críticos, considerando que essa tecnologia é importante para apoiar o desenvolvimento de áreas estratégicas do país, como a de controle do meio ambiente, a de segurança e defesa nacional e a de agricultura. A relação academia-indústria é fortemente explorada no desenvolvimento das soluções nesses diversos domínios, e a disseminação e divulgação científica nessa, e em muitas outras áreas, é ação primordial. Além de outras atividades, deve-se salientar que a ação de divulgação científica está sendo realizada neste momento pela colaboração da Agência de Difusão Científica CienciaWeb do Polo do IEA/SC com grandes benefícios mútuos. Entre as atividades desenvolvidas em parceria com o IEA/CienciaWeb, estão a produção dos programas "Eu Pesquiso", "Quero Saber" e "De Cara Com Feras", palestra no programa CienciaWeb no Ano Internacional da Química e apoio na organização do II Seminário de Jornalismo Científico a ser realizado ainda em 2011.

\section{Sistemas complexos}

Coordenado pelo Prof. Dr. Hamilton Varela, do IQSC/USP. A abordagem reducionista fundamentada na construção do todo a partir da soma das partes tem sido cada vez mais desafiada por problemas postos por diferentes áreas do conhecimento humano. Conceitos como dinâmica não linear, caos, auto-organização dinâmica, padrões espaçotemporais e propriedades emergentes têm sido utilizados e se mostrado úteis tratamentos de problemas oriundos de diferentes campos. Incorporar o conceito de complexidade, ou o arcabouço teórico necessário para tratar os sistemas complexos, pressupõe uma mudança de foco de estruturas, quantidade e reducionismo para padrões, qualidade e visão sistêmica. A capacidade de lidar com o complexo tem se tornado uma necessidade premente no mundo quase plano que vivemos. Questões relativamente recentes, como desenvolvimento sustentável, aquecimento global, globalização e o papel das redes na sociedade, envolvem estruturas hierárquicas em vários níveis e propriedades emergentes, e, portanto, não podem ser discutidas com base nos conceitos reducionistas vigentes. Apesar de corriqueiramente utilizadas em alguns problemas específicos, pode-se afirmar que ainda são poucas as áreas que se beneficiam da estrutura conceitual associada à complexidade. Nesse contexto, a formação de redes nacionais e internacionais tem um papel essencial no processo de entendimento e disseminação da complexidade e, portanto, como uma das possíveis alternativas ao cenário fragmentado e de isolamento que caracteriza o conhecimento no século XXI.

O Grupo de Trabalho em Sistemas Complexos (GTSC) foi fundado em dezembro de 2008 pelos professores Sérgio Mascarenhas, Yvonne Mascarenhas e Hamilton Varela (IQSC/USP). Entre os objetivos gerais do GTSC estão:

a) fomentar a cooperação interdisciplinar entre diferentes grupos de pesquisas básicas e aplicadas, públicas e privadas, do Brasil e do exterior; 
b) estudar, correlacionar e divulgar em diferentes níveis a miríade de aspectos associados ao comportamento complexo em sistemas químicos, físicos, biológicos, sociais e tecnológicos;

c) implantar um portal de difusão e popularização dos conceitos de complexidade em conjunto com a Agência CienciaWeb do IEA-SC;

d) estabelecer uma rede de cooperação internacional com centros de pesquisas e grupos de excelência de diferentes áreas;

e) servir como célula inicial à eventual nucleação de um Centro Internacional em Sistemas Complexos.

Uma das principais contribuições do GTSC no período foi a realização do $1^{\circ}$ Workshop em Sistemas Complexos (do Grupo de Trabalho em Sistemas Complexos do IEA-SC/USP). O evento foi realizado no Instituto de Química de São Carlos, nos dias 3 e 4 de agosto de 2009, e contou com palestrantes de várias áreas do conhecimento e de diferentes instituições de ensino e pesquisa do Brasil. A lista completa dos palestrantes é: Roberto Luzzi (IF/Unicamp), Rita Zorzenon (DF/UFPE), Marcos Buckeridge (IB/USP), Hamilton Varela (IQSC/USP), Ivan Guerrini (Unesp), Marcelo Mazza (IEA-SC/USP), Roberto Berlinck (IQSC/USP), Wanderley dos Santos (IB/USP), Roberto Faria (IQ/UFRJ), Sérgio Mascarenhas (IEA-SC/USP), Roberto Kraenkel (IFT/ Unesp), Gustavo Vilela (IFSC/USP) e Sílvio Crestana (Embrapa). Além desse evento, várias colaborações foram estabelecidas, com destaque para a atuação internacional junto ao "Ertl Center for Electrochemistry and Catalysis" na cidade de Gwangju, Coreia do Sul, e ao Fritz Haber Institute em Berlim, Alemanha. Atualmente, o foco central de atuação do GTSC é a elaboração de uma proposta de criação de um curso de Engenharia de Sistemas Complexos.

\section{IEA-Embrapa}

Coordenado pelo Dr. Paulo Cruvinel, pesquisador do CNPDIA da Embrapa.

As atividades desse GT se concentraram no projeto denominado "Rede de Inovação e Prospecção Tecnológica para o Agronegócio" (Ripa).

O acirramento da concorrência mundial impõe ao agronegócio brasileiro a instrumentalização de saberes que antecipem as ações dos concorrentes e permitam prospectar oportunidades, bem como identificar pontos de estrangulamento. O desafio enfrentado pelos países em desenvolvimento, seja na tentativa de acompanhar a fronteira do desenvolvimento científico, seja para fortalecer seus sistemas regionais ou setoriais de C,T \& I, ou ainda aproveitar as oportunidades geradas pelo avanço do conhecimento científico e tecnológico para o desenvolvimento socioeconômico, é cada vez maior. A maior parte dos países centrais tem aumentado o aporte de recursos públicos e privados dedicados à geração e difusão do conhecimento e à criação e desenvolvimento de redes de cooperação. Essa forma de organização permite flexibilizar áreas de conhecimento já estabelecidas, criando capacidade para mobilizar competências e ativos 
existentes em uma instituição, configurando grupos de trabalho necessários à solução de novos problemas. Desse modo, o compartilhamento de informações e a gestão, em tempo real, dos avanços obtidos por pesquisadores e organizações integrados em redes são apontados como o melhor caminho. De igual modo, deve-se maximizar a utilização comum da infraestrutura física e de recursos para a inovação e prospecção tecnológica. A capacidade de compreender esse ambiente e de vislumbrar novas oportunidades e ameaças ao agronegócio brasileiro está totalmente dependente de um programa sistemático de monitoramento e de análise de dados do ambiente interno e externo e de subsídios que proporcionem uma relativa estabilidade do setor em um ambiente competitivo, o que somente é possível mediante um Sistema de Inteligência Competitiva e Estratégica para o setor.

\section{Objetivos gerais da Ripa}

- realizar estudos avançados e construir uma Rede de Inovação e Prospecção Tecnológica para o Agronegócio;

- criar um ambiente colaborativo que maximize a canalização dos conhecimentos tácitos e explícitos das organizações;

- integrar ações entre instituições do governo, setor produtivo, do terceiro setor e da comunidade de C, T \& I;

- subsidiar o Comitê Gestor do Fundo Setorial de Agronegócio, formuladores de políticas públicas, bem como tomadores de decisão e a Finep no estabelecimento de prioridades e na promoção de iniciativas que pressuponham decisões de natureza estratégica e competitiva, dependentes de inovação e prospecção tecnológica.

O projeto foi concluído e seus resultados podem ser encontrados no site www.ripa.com.br.

\section{Bionanotecnologia}

Coordenado pelo Prof. Sérgio Mascarenhas, com a participação de L. C. Mattoso do Lab. Nac. de Nanotecnologia para o Agronegócio, da Embrapa.

Suas ações levaram à constituição da Rede Nanobiotec-Brasil com apoio da Capes, que hoje apoia 38 sub-redes sobre as varias áreas de interesse em Nanobiologia e Nanobiotecnlogia em todo o Brasil, sendo uma das sub-redes localizada no IFSC, e realiza pesquisas subordinada ao tema "Avanços e riscos da Nanobiotecnologia em medicina" (NanoBioMed). A sub-rede NanoBioMed é coordenada por Yvonne Primerano Mascarenhas e Valtencir Zucolotto, professora titular e professor doutor, respectivamente, do IFSC-USP. Suas atividades são difundidas pelo portal CienciaWeb com especial destaque para entrevistas e resultados apresentados em seu workshop anual.

Com o objetivo de estimular as pesquisas nessa área, esse GT organizou e realizou no Brasil, em 2010, o Primeiro Congresso Internacional em Bionanotecnologia, na cidade de Águas de São Pedro (SP). 


\section{Semicondutores orgânicos}

Coordenado pelo Prof. Roberto Mendonça de Faria, professor titular do IFSC e seu último diretor, atual coordenador desse Polo. Além disso, é atual coordenador do Instituto Nacional de Ciência e Tecnologia do CNPq, denominado Instituto Nacional de Ciência e Tecnologia de Eletrônica Orgânica (Ineo), uma nova área de fronteira na microeletrônica de grande significado para o futuro do país nesse setor estratégico.

O Ineo montou uma rede de pesquisa nacional Instituto Nacional de Ciência e Tecnologia voltada para pesquisas em dispositivos eletrônicos e optoeletrônicos, em que os materiais ativos são filmes de semicondutor orgânico, que podem ser separados em dois tipos principais: polímeros sintéticos e moléculas pequenas, e polímeros naturais. $\mathrm{O}$ nome semicondutor orgânico aqui adotado vem da similaridade em termos de estrutura eletrônica que as moléculas a serem estudadas têm com os semicondutores inorgânicos tradicionais. As atividades do Ineo concentram-se em diferentes classes de materiais orgânicos, foto e/ ou eletroativos. Os projetos de pesquisa que o compõem incluem as áreas de síntese orgânica, estudo de propriedades estruturais, ópticas e elétricas, teoria de transporte em dispositivos e em estrutura eletrônica em nível molecular, processamento e possíveis aplicações de dispositivos.

As atividades do GT no IEA objetivam usar um ambiente propicio à divulgação de suas atividades junto às comunidades acadêmica, aos docentes e estudantes da rede pública de educação e ao público em geral, de modo a estimular a população jovem para as carreiras científicas. Para tanto, utiliza a Agência CienciaWeb existente no Polo de São Carlos do IEA para fazer cobertura de palestras, entrevistas e eventos realizados. Podemos citar, em particular, o Workshop Anual, que conta com a participação de grande parte de seus membros docentes, pós-doc e alunos de mestrado e doutorado.

\section{Agradecimento}

Agradecemos o dedicado esforço de todos os funcionários e ex-funcionários do IEA, Polo de São Carlos, bem como o apoio da Coordenadoria do campus de São Carlos - USP.

Sérgio Mascarenhas é professor titular emérito do IFSC/USP e coordenador de projetos do IEA/USP Polo São Carlos. @ - sergiomascarenhas28@gmail.com

Yvonne Primerano Mascarenhas é professora titular do IFSC/USP e coordenadora administrativa do IEA-USP Polo São Carlos. @ - yvonne@if.sc.usp.br

Recebido em 19.9.2011 e aceito em 25.9.2011. 